\title{
Continuous-variable quantum cryptography with discrete alphabets: Composable security under collective Gaussian attacks
}

\author{
Panagiotis Papanastasiou 1 and Stefano Pirandola \\ Department of Computer Science, University of York, York YO10 5GH, United Kingdom
}

(Received 27 February 2020; accepted 30 July 2020; published 14 January 2021)

\begin{abstract}
We consider continuous-variable quantum key distribution with discrete-alphabet encodings. In particular, we study protocols where information is encoded in the phase of displaced coherent (or thermal) states, even though the results can be directly extended to any protocol based on finite constellations of displaced Gaussian states. In this setting, we study the composable security in the finite-size regime assuming the realistic but restrictive hypothesis of collective Gaussian attacks. Under this assumption, we can efficiently estimate the parameters of the channel via maximum likelihood estimators and bound the corresponding error in the final secret key rate.
\end{abstract}

DOI: 10.1103/PhysRevResearch.3.013047

\section{INTRODUCTION}

Quantum key distribution (QKD) [1-4] allows two remote authenticated parties to establish a shared secret key without any assumption on the computational power of the eavesdropper, the security being based on fundamental laws of quantum mechanics, such as the no-cloning theorem [5,6]. The first QKD protocols were based on the use of discrete variables (DVs), i.e., discrete degrees of freedom of the electromagnetic field, such as polarization or time bins. Later, at the end of the 1990s and beginning of 2000, QKD was extended to continuous-variables (CVs) [7,8] by the work of Ralph [9] and other authors [10-13], culminating in the seminal GG02 protocol [14] based on Gaussian modulation of coherent states. This seminal work also introduced the notion of reverse reconciliation that allowed experimental CV-QKD to reach long distances and led to the theoretical introduction of the reverse coherent information of a bosonic channel $[15,16]$. The authors of Ref. [16] first explored the ultimate limits of pointto-point (i.e., repeaterless) QKD, culminating in 2015 with the discovery and proof of the Pirandola-Laurenza-OttavianiBanchi (PLOB) bound [17] (see Refs. [4,18] for more details on the historical developments).

Other theoretical advances in CV-QKD were the introduction of thermal-state protocols [19-24] (where the authors of Refs. [21,22] specifically studied the extension to longer wavelengths, down to the microwaves), two-way quantum communication protocols [25,26], one-dimensional protocols [27,28], and CV measurement-device independent (MDI) QKD [29]. Last but not least, there was the important development of CV-QKD with discrete-alphabet encoding. This idea was first introduced in the postselection protocol of the authors of Ref. [30] and later developed in a number of works [31-39] . In particular, the authors of Refs. [30-33] considered

Published by the American Physical Society under the terms of the Creative Commons Attribution 4.0 International license. Further distribution of this work must maintain attribution to the author(s) and the published article's title, journal citation, and DOI. binary and ternary alphabets of displaced coherent states. The authors of Ref. [34] considered four coherent states and, later, other works studied alphabets with arbitrary number of states under pure-loss [35] and thermal-loss [36] attacks. All these security proofs were limited to the asymptotic case of infinite signals exchanged by the parties. In particular, the security of discrete-alphabet CV QKD has been proven asymptotically under collective attacks using decoy-like states in Ref. [37] and, more recently, under general attacks using a Gaussian bound in Ref. [38] (see also Ref. [39]).

In this work, we depart from the asymptotic security assumption and study the finite-size composable security of discrete-alphabet CV-QKD protocols. However, this extension comes with the price of another restriction. In fact, our analysis holds under the assumption of collective Gaussian attacks [40] and, in particular, collective entangling cloner attacks $[4,41]$ which results into a realistic thermal-loss channel between the remote parties. While the general arguments apply to any discrete alphabet, we focus on the case of phase-encoded coherent (or thermal) states, so that they are displaced in the phase-space to create regular constellations at fixed distance from the vacuum state. Our techniques combine tools from Refs. [24,42-50]. The assumption of collective Gaussian attack is particularly useful for the purpose of parameter estimation, for which we follow the approach found in Refs. $[24,42,43]$. The composable analysis resorts to various tools as combined in the approach found in Ref. [44] here specified and applied to a discrete-alphabet protocol.

The paper is organized as follows. In Sec. II, we describe the discrete-alphabet (phase-encoded) QKD protocol, for which we discuss the asymptotic security analysis. In Sec. III, we discuss parameter estimation in the presence of finite-size effects and, in Sec. IV, we study the key rate of the protocol in the composable security framework. Section V is for conclusions.

\section{ASYMPTOTIC SECURITY OF A PHASE-ENCODED PROTOCOL}

In a generic phase-encoded CV-QKD protocol with $N$ states, Alice randomly chooses between $N$ coherent states $\left|\alpha_{k}\right\rangle$ 
with amplitude $\alpha_{k}:=2^{-1} \alpha \exp \left(\mathrm{i} 2 k \pi N^{-1}\right)$, where $\alpha \geqslant 0$ and $k=0, \ldots, N-1$ (so that the classical label $k$ is chosen with probability $P_{k}=N^{-1}$ ). More generally, she prepares her mode $A$ in one of $N$ displaced thermal states $\rho_{A \mid k}$ with amplitudes $\alpha_{k}$, each with a fixed mean number of photons $\bar{n}_{\text {th }}$. In terms of quadrature operators $\hat{\mathbf{x}}_{A}:=\left(\hat{q}_{A}, \hat{p}_{A}\right)^{T}$ (with the quantum shot noise equal to 1 ), Alice's conditional thermal state has mean value

$$
\overline{\mathbf{x}}_{A \mid k}:=\operatorname{Tr}\left(\hat{\mathbf{x}}_{A} \rho_{k}\right)=\alpha\left(\begin{array}{c}
\cos \left(2 k \pi N^{-1}\right) \\
\sin \left(2 k \pi N^{-1}\right)
\end{array}\right),
$$

and covariance matrix $(\mathrm{CM}) \mathbf{V}_{A \mid k}=\left(v_{\text {th }}+1\right) \mathbf{I}$, where $v_{\text {th }}=$ $2 \bar{n}_{\text {th }}$ and $\mathbf{I}$ is the bidimensional identity matrix.

The signal state $\rho_{A \mid k}$ is traveling through a Gaussian (thermal-loss) channel which is under the full control of Eve. This is described by transmissivity $\tau$ and injected thermal noise $\omega \geqslant 1$. This channel can always be dilated into an entangling cloner attack [40], where Eve has a two-mode squeezed-vacuum (TMSV) state $\rho_{e E_{0}}$ with zero mean $\overline{\mathbf{x}}_{e E_{0}}=$ $(0,0,0,0)$ and $\mathrm{CM}$

$$
\mathbf{V}_{e E_{0}}=\left(\begin{array}{cc}
\omega \mathbf{I} & \sqrt{\omega^{2}-1} \mathbf{Z} \\
\sqrt{\omega^{2}-1} \mathbf{Z} & \omega \mathbf{I}
\end{array}\right),
$$

where $\mathbf{Z}=\operatorname{diag}\{1,-1\}$. In particular, mode $e$ is mixed with Alice's traveling mode $A$ in a beam-splitter with transmissivity $\tau$ described by the symplectic matrix

$$
\mathcal{B}(\tau)=\left(\begin{array}{cc}
\sqrt{\tau} \mathbf{I} & \sqrt{1-\tau} \mathbf{I} \\
-\sqrt{1-\tau} \mathbf{I} & \sqrt{\tau} \mathbf{I}
\end{array}\right) .
$$

After the interaction, modes $e^{\prime}$ and $E_{0}$ are kept in a quantum memory for an optimal final measurement taking into consideration all the classical communication between the parties. For each use of the channel, Eve's and Bob's conditional output state $\rho_{B e^{\prime} E_{0} \mid k}$ has mean value and $\mathrm{CM}$ given by

$$
\begin{aligned}
\overline{\mathbf{x}}_{B e^{\prime} E_{0} \mid k}=[\mathcal{B}(\tau) \oplus \mathbf{I}]\left(\overline{\mathbf{x}}_{A \mid k} \oplus \overline{\mathbf{x}}_{e E_{0}}\right)=\overline{\mathbf{x}}_{B \mid k} \oplus \overline{\mathbf{x}}_{e^{\prime} E_{0} \mid k}, \\
\mathbf{V}_{B e^{\prime} E_{0} \mid k}=[\mathcal{B}(\tau) \oplus \mathbf{I}]\left(\mathbf{V}_{A \mid k} \oplus \mathbf{V}_{e E_{0}}\right)\left[\mathcal{B}(\tau)^{\top} \oplus \mathbf{I}\right] \\
=\left(\begin{array}{cc}
\mathbf{B} & \mathbf{C} \\
\mathbf{C}^{\top} & \mathbf{V}_{e^{\prime} E_{0} \mid k}
\end{array}\right) .
\end{aligned}
$$

At the output, assume that Bob applies heterodyne measurement with outcome $\left(q_{B}, p_{B}\right)$. Then, Eve's triply conditional state $\rho_{e^{\prime} E_{0} \mid k q_{B} p_{B}}$ has mean value and CM [51-53]

$$
\begin{gathered}
\overline{\mathbf{x}}_{e^{\prime} E_{0} \mid q_{B} p_{B} k}=\overline{\mathbf{x}}_{e^{\prime} E_{0} \mid k}-\mathbf{C}^{\top}(\mathbf{B}+\mathbf{I})^{-1}\left[\overline{\mathbf{x}}_{B \mid k}-\left(\begin{array}{c}
q_{B} \\
p_{B}
\end{array}\right)\right], \\
\mathbf{V}_{e^{\prime} E_{0} \mid q_{B} p_{B} k}=\mathbf{V}_{e^{\prime} E_{0} \mid k}-\mathbf{C}^{\top}(\mathbf{B}+\mathbf{I})^{-1} \mathbf{C},
\end{gathered}
$$

while the probability of the outcome is given by

$$
P_{q_{B} p_{B} \mid k}=\frac{e^{-\frac{1}{2} \frac{\left[q_{B}-\sqrt{\tau} \alpha \cos \left(2 k \pi N^{-1}\right)\right]^{2}+\left[p_{B}-\sqrt{\tau} \alpha \sin \left(2 k \pi N^{-1}\right)\right]^{2}}{\Omega}}}{2 \pi \Omega},
$$

with $\Omega:=2+\tau v_{\text {th }}+(1-\tau)(\omega-1)$. Setting

$$
q_{B}+\mathrm{i} p_{B}=\beta e^{\mathrm{i}\left(2 l \pi N^{-1}+\theta\right)},
$$

with $\beta \geqslant 0$ and $\theta \in\left[-\pi N^{-1}, \pi N^{-1}\right]$, we obtain

$$
\begin{aligned}
P_{\beta \theta l \mid k}= & \frac{1}{2 \pi \Omega} e^{\frac{-\left[\beta \cos \left(2 l \pi N^{-1}+\theta\right)-\sqrt{\tau} \alpha \cos \left(2 l \pi N^{-1}\right)\right]^{2}}{2 \Omega}} \\
& \times e^{\frac{-\left[\beta \sin \left(2 l \pi N^{-1}+\theta\right)-\sqrt{\tau} \alpha \sin \left(2 l \pi N^{-1}\right)\right]^{2}}{2 \Omega}} .
\end{aligned}
$$

Integrating over for $\beta$ and for $\theta$, we derive

$$
P_{l \mid k}=\iint_{0,-\pi N^{-1}}^{\infty, \pi N^{-1}} \beta P_{\beta \theta l \mid k} d \beta d \theta,
$$

which can be calculated numerically. Here $l$ is Bob's estimator of Alice's encoding variable $k$. Using Bayes' formula we may write

$$
P_{k \mid l}=\frac{P_{l \mid k} P_{k}}{\sum_{k=0}^{N-1} P_{k} P_{l \mid k}},
$$

and compute the residual entropy

$$
H(k \mid l)=\sum_{l} P_{l} \sum_{k}\left(-P_{k \mid l} \log _{2} P_{k \mid l}\right) .
$$

The mutual information between the variables $k$ and $l$ is given by

$$
I(k: l)=H(k)-H(k \mid l)=\log _{2} N-H(k \mid l) .
$$

In reverse reconciliation (RR), Eve's information on $l$ is bounded by the Holevo quantity

$$
\chi(E: l)=S\left(\rho_{E}\right)-\sum_{l} P_{l} S\left(\rho_{E \mid l}\right)
$$

with $E:=e^{\prime} E_{0}$, where $\rho_{E}:=\sum_{l} P_{l} \rho_{E \mid l}$ is non-Gaussian, and the conditional state $\rho_{E \mid l}$ is calculated by using the replacement of Eq. (9) in the Gaussian state $\rho_{E \mid q_{B} p_{B} k}$ [54] and averaging over the probability $P_{k \beta \theta \mid l}$, i.e., we have

$$
\rho_{E \mid l}=\sum_{k=0}^{N-1} \iint_{0,-\pi N^{-1}}^{\infty, \pi N^{-1}} P_{\beta \theta k \mid l} \rho_{E \mid \beta \theta l k} d \theta d \beta,
$$

where

$$
P_{\beta \theta k \mid l}=\frac{P_{\beta \theta l \mid k} P_{k}}{P_{l}} .
$$

Thus, the asymptotic secret key rate in RR is given by [55]

$$
R=\xi I(k: l)-\chi(E: l),
$$

where $\xi \in[0,1]$ is the reconciliation efficiency. In Fig. 1, we plotted this rate (solid black line) for the case of two states $(N=2)$ with $\xi=1$, assuming excess noise $\varepsilon:=\tau^{-1}(1-$ $\tau)(\omega-1)=0.01$ and setting $\alpha=2$. In Fig. 2, we showed the corresponding rate for $N=3$, assuming the same parameters.

\section{CHANNEL PARAMETER ESTIMATION}

The asymptotic rate in Eq. (18) is a function of Alice's encoding parameters, i.e., $\alpha, N$, and $\nu_{\text {th }}$, together with the channel parameters, i.e., $\tau$ and $\omega$, or equivalently $\tau$ and $\varepsilon$. To estimate the parameters of the channel, Alice and Bob sacrifice $m$ signal states. By communicating their outcomes for these $m$ signals, Alice and Bob can compute estimators for $\tau$ and $V_{\varepsilon}:=\tau \varepsilon$, and corresponding confidence intervals. 


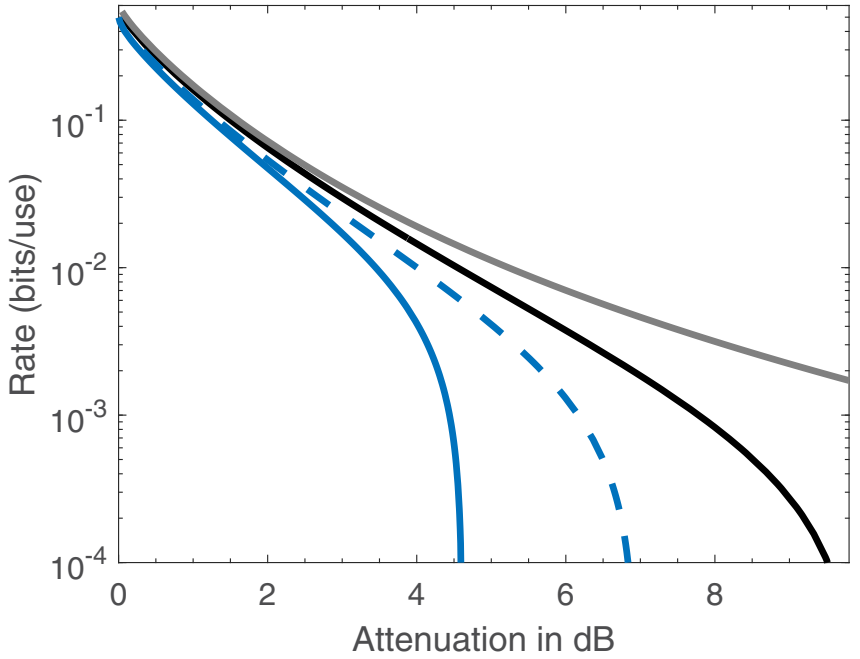

FIG. 1. Secret key rate for $N=2$ versus attenuation in $\mathrm{dB}$. We assume $\alpha=2$ and excess noise $\varepsilon=0.01$. We show the asymptotic case with $\xi=1$ (black solid line) and the composable case, for which we assume $\epsilon_{\mathrm{s}}=\epsilon_{\mathrm{h}}=10^{-10}, \epsilon_{\mathrm{PE}}=10^{-10}, p=0.9, \xi=0.99$, and $r=0.01$, for $M=10^{12}$ (blue dashed line) and $M=10^{9}$ (blue solid line). All the lines have a truncation accuracy of 10 Fock-basis states. For comparison, we also plot the corresponding asymptotic rate (gray solid line) assuming a pure loss channel.

They can choose worst-case parameters to be used in the computation of the key rate in Eq. (18).

Therefore, assume that Alice reveals the encoding $k$ of $m$ signal states out of a block of $M=m+n$ signal states. For $m$ sufficiently large, we have that $m / N$ can be chosen to be an integer. Bob will have samples $B_{k i}$ for $i=1, \ldots, m / N$

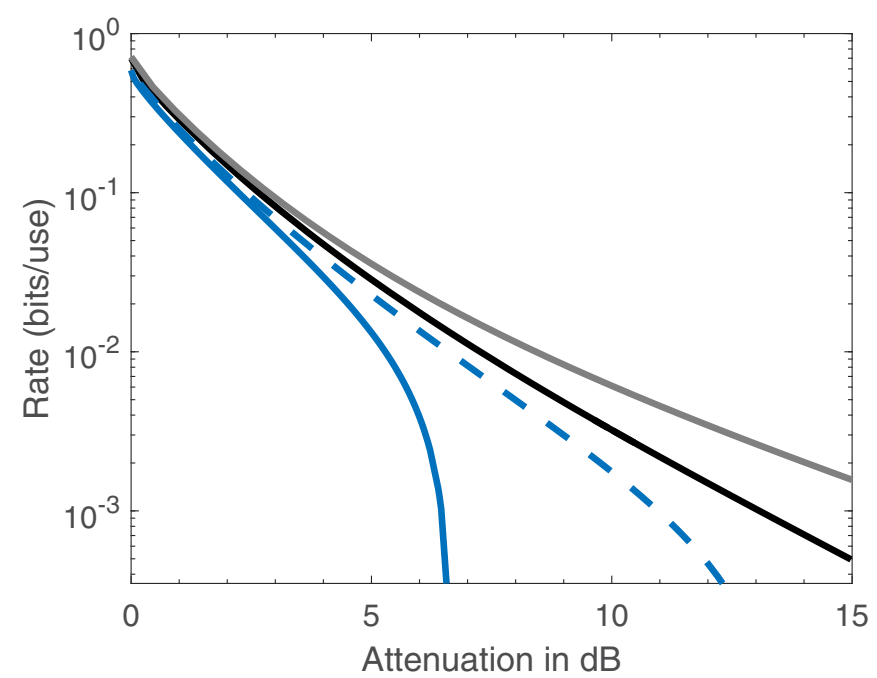

FIG. 2. The secret key rate for $N=3$ versus attenuation in $\mathrm{dB}$. We assume $\alpha=2$ and excess noise $\varepsilon=0.01$. We include the asymptotic case with $\xi=1$ (black solid line) and the composable case, for which we assume $\epsilon_{\mathrm{s}}=\epsilon_{\mathrm{h}}=10^{-10}, \epsilon_{\mathrm{PE}}=10^{-10}, p=0.9, \xi=0.99$, and $r=0.01$, for $M=10^{12}$ (blue dashed line) and $M=10^{9}$ (blue solid line). All the lines have a truncation accuracy of 10 Fock-basis states. For comparison, we also plot the corresponding asymptotic rate (gray solid line) assuming a pure loss channel. associated to a specific Alice's encoding $k$. Because we assume heterodyne detection, the discussion of the $\hat{q}$ and $\hat{p}$ quadratures is symmetric. In the $\hat{q}$ quadrature, Bob's sampled $q$-quadratures $B_{k i}$ can be described by the following stochastic variable:

$$
\begin{gathered}
q_{B_{k}}=\sqrt{\frac{\tau}{2}} \alpha \cos (2 k \pi / N)+q_{\mathrm{no}}, \\
q_{\mathrm{no}}:=\sqrt{\frac{\tau}{2}} q_{\mathrm{th}}+\sqrt{\frac{1-\tau}{2}} q_{E}+\sqrt{\frac{1}{2}} q_{\mathrm{h}},
\end{gathered}
$$

where $q_{\mathrm{th}}$ is Alice preparation noise with variance $v_{\text {th }}+1, q_{E}$ is Eve's noise variable with variance $\omega$, and $q_{\mathrm{h}}$ is the noise variable due to Bob's heterodyne measurement. The variable $q_{B_{k}}$ is Gaussian with mean

$$
\mathbb{E}\left(q_{B_{k}}\right)=\sqrt{\frac{\tau}{2}} \alpha \cos (2 k \pi / N),
$$

and variance

$$
V_{\mathrm{no}}=\frac{1}{2} \Omega=\frac{1}{2}\left(\tau v_{\mathrm{th}}+V_{\varepsilon}+2\right) .
$$

We can then create maximum likelihood estimators for the mean value and variance of $q_{B_{k}}$ starting from the samples $B_{k i}$. In fact, we may write

$$
\widehat{\overline{q_{B_{k}}}}=\frac{N}{m} \sum_{i=1}^{m / N} B_{k i}, \quad \widehat{V}_{\mathrm{no}_{k}}=\frac{N}{m} \sum_{i=1}^{m / N}\left(B_{k i}-\widehat{\bar{q}_{B_{k}}}\right)^{2} .
$$

The mean value and variance of the estimator $\widehat{\bar{q}_{B_{k}}}$ are given by

$$
\begin{gathered}
\mathbb{E}\left(\widehat{\overline{q_{B_{k}}}}\right)=\frac{N}{m} \sum_{i=1}^{m / N} \mathbb{E}\left(B_{k i}\right)=\mathbb{E}\left(q_{B_{k}}\right), \\
\operatorname{Var}\left(\widehat{\bar{q}_{B_{k}}}\right)=\frac{N^{2}}{m^{2}} \sum_{i=1}^{m / N} \operatorname{Var}\left(B_{k i}\right)=\frac{N}{m} V_{\mathrm{no}},
\end{gathered}
$$

since $B_{k i}$ can be considered to be i.i.d. variables (in a collective Gaussian attack).

Note that the estimator $\widehat{\widehat{q_{B_{k}}}}$ can be replaced by its expected value $\mathbb{E}\left(q_{B_{k}}\right)$ due to the fact that its variance in Eq. (25) vanishes for $m \gg 1$. Thus, we can write the variance estimator $\widehat{V}_{\text {no } k}$ in Eq. (23) as

$$
\widehat{V}_{\mathrm{no}_{k}}=V_{\mathrm{no}} \frac{N}{m} \sum_{i=1}^{m / N}\left(\frac{B_{k i}-\mathbb{E}\left(q_{B_{k}}\right)}{\sqrt{V_{\mathrm{no}}}}\right)^{2} .
$$

The term inside the brackets follows a standard normal distribution with zero mean and unit variance. Therefore, the sum term follows a noncentral chi-squared distribution with mean equal to $m / N$ and variance $2 m / N$. Consequently, for the mean and variance of the estimator $\widehat{V}_{\mathrm{no}_{k}}$ we obtain

$$
\begin{gathered}
\mathbb{E}\left[\widehat{V}_{\mathrm{no}_{k}}\right]=V_{\mathrm{no}} \frac{N}{m} \mathbb{E}\left[\sum_{i=1}^{m / N}\left(\frac{B_{k i}-\mathbb{E}\left(q_{B_{k}}\right)}{\sqrt{V_{\mathrm{no}}}}\right)^{2}\right]=V_{\mathrm{no}}, \\
\operatorname{Var}\left[\widehat{V}_{\mathrm{no}_{k}}\right]=V_{\mathrm{no}}^{2}\left(\frac{N}{m}\right)^{2} \operatorname{Var}\left[\sum_{i=1}^{m / N}\left(\frac{B_{k i}-\mathbb{E}\left(q_{B_{k}}\right)}{\sqrt{V_{\mathrm{no}}}}\right)^{2}\right]=2 \frac{N}{m} V_{\mathrm{no}}^{2} .
\end{gathered}
$$


Based on the estimator $\widehat{\bar{q}_{B_{k}}}$ we can build an estimator for the transmissivity [cf. Eq. (19)]

$$
\hat{\tau}_{k}=2 \alpha^{-2} \cos ^{-2}(2 k \pi / N)\left(\widehat{\bar{q}_{B_{k}}}\right)^{2} .
$$

The estimator $\widehat{\bar{q}_{B_{k}}}$ is the sample mean of $B_{k i}$ and as such follows a Gaussian distribution. We then can express Eq. (29) with the help of the noncentral chi-squared variable $\chi_{k} \equiv$ $\left(\sqrt{\frac{m}{N}} \frac{\widehat{\bar{q}_{B_{k}}}}{\sqrt{V_{\mathrm{no}}}}\right)^{2}$ as follows:

$$
\hat{\tau}_{k}=2 \frac{V_{\mathrm{no}}}{[\alpha \cos (2 k \pi / N)]^{2}} \frac{N}{m}\left(\sqrt{\frac{m}{N}} \frac{\widehat{\bar{q}_{B_{k}}}}{\sqrt{V_{\mathrm{no}}}}\right)^{2} .
$$

Because $\chi_{k}$ has mean value $1+\frac{m}{N} \frac{\tau[\alpha \cos (2 k \pi / N)]^{2}}{2 V_{\mathrm{no}}}$ and variance $2\left(1+2 \frac{m}{N} \frac{\tau[\alpha \cos 2 k \pi / N]^{2}}{2 V_{\text {no }}}\right)$, the estimator of the transmissivity has mean and variance equal to

$$
\begin{aligned}
\mathbb{E}\left(\hat{\tau}_{k}\right)= & \frac{2 V_{\mathrm{no}} N}{m \alpha^{2} \cos ^{2}(2 k \pi / N)} \\
& \times\left(1+\frac{m}{N} \frac{\tau[\alpha \cos (2 k \pi / N)]^{2}}{2 V_{\mathrm{no}}}\right) \\
= & \tau+\mathcal{O}(1 / m), \\
\operatorname{Var}\left(\hat{\tau}_{k}\right):= & \sigma_{k}^{2}=\left(\frac{2 V_{\mathrm{no}} N}{m \alpha^{2} \cos ^{2}(2 k \pi / N)}\right)^{2} \\
& \times 2\left(1+2 \frac{m}{N} \frac{\tau[\alpha \cos (2 k \pi / N)]^{2}}{2 V_{\mathrm{no}}}\right) \\
= & 8 \tau \frac{N}{m} \frac{V_{\mathrm{no}}}{\alpha^{2} \cos ^{2}(2 k \pi / N)}+\mathcal{O}\left(1 / m^{2}\right) .
\end{aligned}
$$

Since there will be other estimators corresponding to the other values of Alice's encoding $k$, we can create an optimal linear combination of them with variance [43]

$$
\begin{aligned}
\sigma_{q}^{2} & =\left[\sum_{k=0}^{N-1}\left(\sigma_{k}^{2}\right)^{-1}\right]^{-1} \\
& =8 \tau \frac{N}{m} \frac{V_{\mathrm{no}}}{\alpha^{2}}\left[\sum_{k=0}^{N-1} \cos (2 k \pi / N)\right]^{-1} \\
& =\tau \frac{16}{m} \frac{V_{\mathrm{no}}}{\alpha^{2}} .
\end{aligned}
$$

So far, we used only samples from the $q$-quadrature of Bob's outcomes. Similar relations will hold for the $p$ quadrature. Combining all the available $q$ - and $p$-samples, the optimal linear estimator $\hat{\tau}$ of the transmissivity will have

$$
\mathbb{E}(\hat{\tau})=\tau, \quad \operatorname{Var}(\hat{\tau}):=\sigma^{2}=\tau \frac{8}{m} \frac{V_{\mathrm{no}}}{\alpha^{2}} .
$$

In fact, for large $m$, we can approximate all the $2 N$ estimators $\hat{\tau}_{k}$ to have Gaussian distributions with the same mean and variance $\sigma_{p}^{2}=\sigma_{q}^{2}$. As a result, the global estimator $\hat{\tau}$ is a Gaussian variable with the same mean $\tau$ and variance equal to $\sigma^{2}$. Now, assuming an error $\epsilon_{P E}=10^{-10}$ for channel parameter estimation (PE), we have to consider a 6.5 standard deviation interval for $\tau$. This means that the worst-case value for the transmissivity is equal to

$$
\tau^{\epsilon_{\mathrm{PE}}}=\tau-6.5 \sqrt{\tau \frac{8}{m} \frac{V_{\mathrm{no}}}{\alpha^{2}}} .
$$

Starting from $\widehat{V}_{\mathrm{no}_{k}}$ we may also define an estimator for the excess noise. Solving Eq. (22) with respect to $V_{\varepsilon}$, we obtain

$$
\widehat{V}_{\varepsilon_{k}}=2 \widehat{V}_{\mathrm{no}_{k}}-\hat{\tau} \nu_{\mathrm{th}}-2 \text {. }
$$

Then the mean and variance of this estimator are given by

$$
\begin{gathered}
\mathbb{E}\left[\widehat{V}_{\varepsilon_{k}}\right]=2 V_{\text {no }}-\tau v_{\text {th }}-2, \\
\operatorname{Var}\left[\widehat{V}_{\varepsilon_{k}}\right]:=s_{k}^{2}=8 \frac{N}{m} V_{\text {no }}^{2}+\sigma^{2} v_{\text {th }}^{2},
\end{gathered}
$$

where we used Eqs. (27), (28), and (34). The variance of the optimal linear combination $\widehat{V}_{\varepsilon}$ of all the estimators $\widehat{V}_{\varepsilon_{k}}$ (also considering the $p$-quadrature) is given by

$$
s^{2}=\frac{4 V_{\mathrm{no}}^{2}}{m}+\frac{\sigma^{2} v_{\mathrm{th}}^{2}}{2 N} .
$$

Based on the assumption of large $m$, we approximate the distribution of each $\widehat{V}_{\mathrm{no}_{k}}$ to be Gaussian. As a result, the distribution of $\widehat{V}_{\varepsilon}$ is Gaussian with the same mean and variance given by $s^{2}$ above. Assuming an error $\epsilon_{P E}=10^{-10}$, we obtain the 6.5 confidence intervals for $\widehat{V}_{\varepsilon}$. Therefore, the worst-case value is give by

$$
V_{\varepsilon}^{\epsilon_{\mathrm{PE}}}=V_{\varepsilon}+6.5 \sqrt{\frac{4 V_{\mathrm{no}}^{2}}{m}+\frac{\sigma^{2} v_{\mathrm{th}}^{2}}{2 N}} .
$$

Using the worst-case values $\tau^{\epsilon_{\mathrm{PE}}}$ and $V_{\varepsilon}^{\epsilon_{\mathrm{PE}}}$, we can write a finite-size expression of the key rate $R=R\left(\tau, V_{\varepsilon}\right)$ of Eq. (18) which accounts for the imperfect parameter estimation and the reduced number of signals. This is given by replacing

$$
R\left(\tau, V_{\varepsilon}\right) \rightarrow \frac{n}{M} R\left(\tau^{\epsilon \mathrm{PE}}, V_{\varepsilon}^{\epsilon \mathrm{PE}}\right):=\frac{n}{M} R_{\epsilon_{\mathrm{PE}}} .
$$

\section{COMPOSABLE SECURITY UNDER COLLECTIVE ATTACKS}

The following study for the composable security is based on various ingredients [44-50]. More precisely, it follows the procedure formulated in Ref. [44], which is here specified and applied to a discrete alphabet.

After the parties exchange $n$ signal states and apply error correction (EC), they share a state $\tilde{\rho}^{n}$ from which, according to the leftover hash lemma, they can extract $s_{n}$ bits of uniform randomness, or in other words secret key bits. This number of bits is bounded according to the following relation $[47,48]$ :

$$
s_{n} \geqslant H_{\min }^{\epsilon_{\mathrm{s}}}\left(l^{n} \mid E^{n}\right)_{\tilde{\rho}^{n}}+2 \log _{2} \sqrt{2} \epsilon_{\mathrm{h}}-\operatorname{leak}_{n, \mathrm{EC}}\left(n, \epsilon_{\mathrm{cor}}\right) .
$$

Here, $H_{\text {min }}^{\epsilon_{\mathrm{s}}}\left(l^{n} \mid E^{n}\right)$ is the smooth min-entropy of Bob's variable $l$ conditioned on Eve's systems $E$, and $\operatorname{leak}_{\mathrm{EC}}\left(n, \epsilon_{\mathrm{cor}}\right)$ is the classical information exchanged by the parties for EC (stored by Eve in her register).

The uniform randomness $\epsilon_{\mathrm{h}}$ and smoothing $\epsilon_{\mathrm{s}}$ parameters define the secrecy of the protocol $\epsilon_{\mathrm{sec}}=\epsilon_{\mathrm{h}}+\epsilon_{\mathrm{s}}$ which, along with the EC parameter $\epsilon_{\text {cor }}$, defines the security parameter $\epsilon_{\mathrm{tot}}=\epsilon_{\mathrm{cor}}+\epsilon_{\mathrm{sec}}$. The later bounds the trace distance $D$ of the state $\bar{\rho}^{n}$ (after privacy amplification) from the ideal output 
state $\rho_{\text {id }}$ of a QKD protocol, i.e., a classical-quantum state where the uniformly distributed classical registers of Alice and Bob are uncorrelated from Eve's systems [50]. Each of the epsilon parameters introduced above can be considered to be very small. We take them of the order of $10^{-10}$.

Equation (42) can be further simplified so as to be connected with the asymptotic secret key rate. In fact, we can further bound the smooth min-entropy calculated in terms of $\tilde{\rho}^{n}$ with the smooth min-entropy of the state before EC $\rho^{\otimes n}$, which is in a tensor-product form due to the fact that we assumed a collective attack. More precisely, we exploit the following inequality [44]:

$$
H_{\mathrm{min}}^{\epsilon_{\mathrm{s}}}\left(l^{n} \mid E^{n}\right)_{\tilde{\rho}^{n}} \geqslant H_{\mathrm{min}}^{p \epsilon_{\mathrm{s}}^{2} / 3}\left(l^{n} \mid E^{n}\right)_{\rho^{\otimes n}}+\log _{2}\left[p\left(1-\epsilon_{\mathrm{s}}^{2} / 3\right)\right] .
$$

Here $p$ is the probability of successful EC, i.e., the probability that the protocol is not aborted after Alice and Bob compared hashes of their sequences [4]. The value of $1-p$ is given by the experimental frame error rate [56]. Note that, even if the protocol does not abort (because the hashes are the same), Alice's and Bob's sequences are identical up to an error probability $\epsilon_{\mathrm{cor}}$.

The replacement in Eq. (43) allows us to use the asymptotic equipartition property (AEP) theorem [49] so as to reduce the conditional smooth-min entropy of the tensor-product form $\rho^{\otimes n}$ to the conditional von Neumann entropy $S(l \mid E)_{\rho}$ of the single copy $\rho$. In particular, one may write the following [48]:

$$
H_{\mathrm{min}}^{p \epsilon_{\mathrm{s}}^{2} / 3}\left(l^{n} \mid E^{n}\right)_{\rho^{\otimes n}} \geqslant n S(l \mid E)_{\rho}-\sqrt{n} \Delta_{\mathrm{AEP}}\left(p \epsilon_{\mathrm{s}}^{2} / 3,|\mathcal{L}|\right),
$$

where

$$
\Delta_{\mathrm{AEP}}\left(\epsilon_{\mathrm{s}},|\mathcal{L}|\right):=4 \log _{2}(2 \sqrt{|\mathcal{L}|}+1) \sqrt{\log \left(2 / \epsilon_{\mathrm{s}}^{2}\right)} .
$$

The parameter $|\mathcal{L}|$ is the cardinality of Bob's outcome (alphabet) and, in our case, it is equal to $N$.

Replacing Eqs. (43) and (44) in Eq. (42), one obtains the following bound for the number of secret bits:

$$
\begin{aligned}
s_{n} \geqslant & n S(l \mid E)_{\rho}-\sqrt{n} \Delta_{\mathrm{AEP}}\left(p \epsilon_{\mathrm{s}}^{2} / 3, N\right) \\
& +\log _{2}\left[p\left(1-\epsilon_{\mathrm{s}}^{2} / 3\right)\right] \\
& +2 \log _{2} \sqrt{2} \epsilon_{\mathrm{h}}-\operatorname{leak}_{\mathrm{EC}}\left(n, \epsilon_{\mathrm{cor}}\right) .
\end{aligned}
$$

To further simplify the bound above, consider the definition of quantum mutual information between two systems $Q$ and $E$ in terms of the (conditional) von Neumann entropy

$$
I(Q: E)=S(Q)-S(Q \mid E) .
$$

When $Q$ is a classical system described by a variable $l, I(l$ : $E)$ takes the form of the Holevo information $\chi(E: l)$ and the von Neumann entropy simplifies to the Shannon entropy $H(l)$. Thus we can write

$$
S(l \mid E)_{\rho}=H(l)_{\rho}-\chi(E: l)_{\rho} .
$$

Moreover, let us set the quantity

$$
H(l)_{\rho}-n^{-1} \operatorname{leak}_{\mathrm{EC}}\left(n, \epsilon_{\mathrm{cor}}\right):=\xi I(k: l)_{\rho},
$$

where $I(k: l)$ is the classical mutual information between Alice's and Bob's variables and $\xi \in[0,1]$ defines the reconciliation efficiency [57]. As a result, the asymptotic secret key rate of Eq. (18) appears if we make the previous replacements in Eq. (46) obtaining

$$
\begin{aligned}
s_{n} \geqslant & n R_{\rho}-\sqrt{n} \Delta_{\mathrm{AEP}}\left(p \epsilon_{\mathrm{s}}^{2} / 3, N\right) \\
& +\log _{2}\left[p\left(1-\epsilon_{\mathrm{s}}^{2} / 3\right)\right]+2 \log _{2} \sqrt{2} \epsilon_{\mathrm{h}} .
\end{aligned}
$$

Finally, let us account for the PE in the bound above. This means that we need to write Eq. (50) considering the worstcase scenario state $\rho_{\epsilon_{\mathrm{PE}}}^{M-m}$, where the channel parameters $\tau$ and $V_{\varepsilon}$ are bounded by $\tau^{\epsilon_{\mathrm{PE}}}$ and $V_{\varepsilon}^{\epsilon_{\mathrm{PE}}}$, and also accounting for the fact that we sacrificed $m$ out of $M$ signal states. Therefore, we obtain

$$
\begin{aligned}
s_{M-m} \geqslant & (M-m) R_{\epsilon_{\mathrm{PE}}}-\sqrt{M-m} \Delta_{\mathrm{AEP}}\left(p \epsilon_{\mathrm{s}}^{2} / 3, N\right) \\
& +\log _{2}\left[p\left(1-\epsilon_{\mathrm{s}}^{2} / 3\right)\right]+2 \log _{2} \sqrt{2} \epsilon_{\mathrm{h}},
\end{aligned}
$$

where $R_{\epsilon \mathrm{PE}}$ is the finite-size rate of Eq. (41). This is true only with probability $1-\epsilon_{\mathrm{PE}}$ since there is a nonzero probability $\epsilon_{\mathrm{PE}}$ that the actual values of the channel parameters are not bounded by $\tau^{\epsilon_{\mathrm{PE}}}$ and $V_{\varepsilon}^{\epsilon_{\mathrm{PE}}}$. Dividing Eq. (51) by the total number $M$ of signal states, multiplying by the EC success probability $p$, and setting $r=\frac{m}{M}$, we obtain the secret key rate

$$
\begin{aligned}
R_{M, r} & \geqslant(1-r) p\left[R_{\epsilon_{\mathrm{PE}}}-\frac{1}{\sqrt{(1-r) M}} \Delta_{\mathrm{AEP}}\left(p \epsilon_{\mathrm{s}}^{2} / 3, N\right)\right. \\
& \left.+\frac{\log _{2}\left[p\left(1-\epsilon_{\mathrm{s}}^{2} / 3\right)\right]+2 \log _{2} \sqrt{2} \epsilon_{\mathrm{h}}}{(1-r) M}\right],
\end{aligned}
$$

which is valid up to an overall $\epsilon_{\mathrm{tot}}=\epsilon_{\mathrm{cor}}+\epsilon_{\mathrm{s}}+\epsilon_{\mathrm{h}}+p \epsilon_{\mathrm{PE}}$. See Ref. [44] for corresponding analytical formulas but in the setting of Gaussian-modulated protocols.

In Fig. 1, we plot the composable key rate for the protocol with two states $(N=2)$ versus the attenuation in $\mathrm{dB}$ for $r=0.01, M=10^{12}$ (blue dashed line) and $M=10^{9}$ (blue solid line). We assume excess noise $\varepsilon=0.01$ and set the security parameters to $\epsilon_{\mathrm{s}}=\epsilon_{\mathrm{h}}=\epsilon_{\mathrm{PE}}=10^{-10}$. We assume that the reconciliation efficiency parameter is $\xi=0.99$ and the EC success probability is $p=0.9$. (Note that, in our analysis the EC error $\epsilon_{\text {cor }}$ is contained in $\xi$.) In Fig. 2, we plot the secret key rate for $N=3$, both in the asymptotic (black line) and the composable cases (blue lines) for channel excess noise $\varepsilon=0.01$. As expected, the performance of the protocol is dependent on the number $M$ of signals.

As we can see in Fig. 3, increasing preparation (trusted) thermal noise $v_{\text {th }}$ [23], the fidelity of the signal states increases, making them more difficult to distinguish, resulting in a better secret key rate performance. In more detail, we observe that the fidelity (computed according to Ref. [61]) reaches a saturation point faster when $\alpha$ is smaller. Furthermore, in this point the fidelity becomes closer to 1 as $\alpha$ gets smaller. Taking into consideration the channel propagation, this leads to a configuration where Bob's states may have almost the initial fidelity, while the fidelity of Eve's states may be at the saturation point. This can happen for example for transmissivities that are close to 1 . An additional optimal value of the thermal preparation noise can boost this effect for other transmissivities. In fact, we can observe this in Fig. 4, where we consider excess noise $\varepsilon=0.01$ and preparation noise $v_{\text {th }}=0.1$, i.e., Alice sending thermal states. We observe 


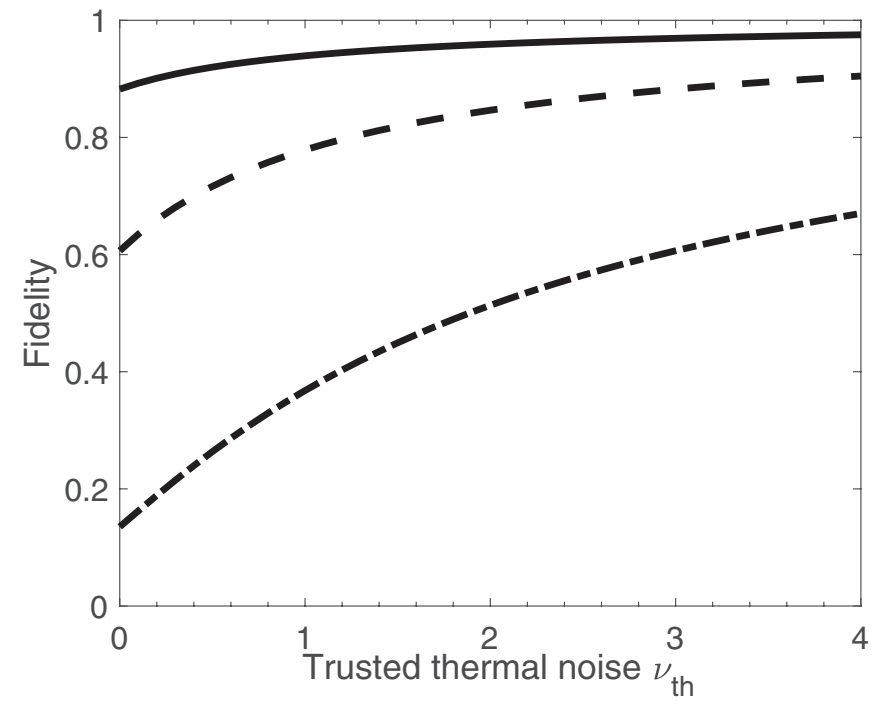

FIG. 3. The fidelity between two signal states $(N=2)$ for $k=0$ and $k=1$ versus the thermal preparation noise $v_{\text {th }}$. We include plots for different amplitudes $\alpha=0.5$ (solid line), $\alpha=1$ (dashed line), and $\alpha=2$ (dashed-dotted line). As the thermal noise increases, the fidelity between the two states arrives at a saturation point close to 1 . The smaller the value of $\alpha$ the faster this saturation happens.

an advantage for the secret key rate when we use preparation noise that compensates the rate degradation due to the finitesize effects.

\section{CONCLUSION AND DISCUSSION}

In this work, we study the finite-size composable security of a discrete-alphabet CV-QKD protocol under the assumption of collective Gaussian attacks. This assumption is realistic because the standard model of loss and noise in optical quantum communications is the memoryless thermal-loss channel, which is dilated into a collective entangling cloner attack, i.e., a specific type of collective Gaussian attack [40]. Our analysis extends previous asymptotic analyses [38,39] to the finite-size and composable regime, but simultaneously pays the price to be restricted to collective Gaussian attacks. Removing this assumption is the subject of future investigations.

Since our analysis applies not only to displaced coherent states but also to displaced thermal states, it can be useful for studying the security of phase-encoded protocols at frequencies lower than the optical. Moreover, the use of displaced thermal states can increase the difficulty in distinguishing the signal states with a beneficial effect for the secret key rate. It is also worth stressing that our derivation, described for phase-encoded signals, can immediately be extended to any constellation of displaced Gaussian states (e.g., coherent, thermal, or squeezed). The most crucial part is the finite-size rate $R_{\epsilon \mathrm{PE}}$ which can always be estimated, under the assumption of collective Gaussian attacks, by using maximum likelihood estimators and their confidence intervals, i.e., adopting simple variations of the technique in Sec. III. In this way, the finite-size rate $R_{\epsilon_{\mathrm{PE}}}$ can always be expressed in terms of the

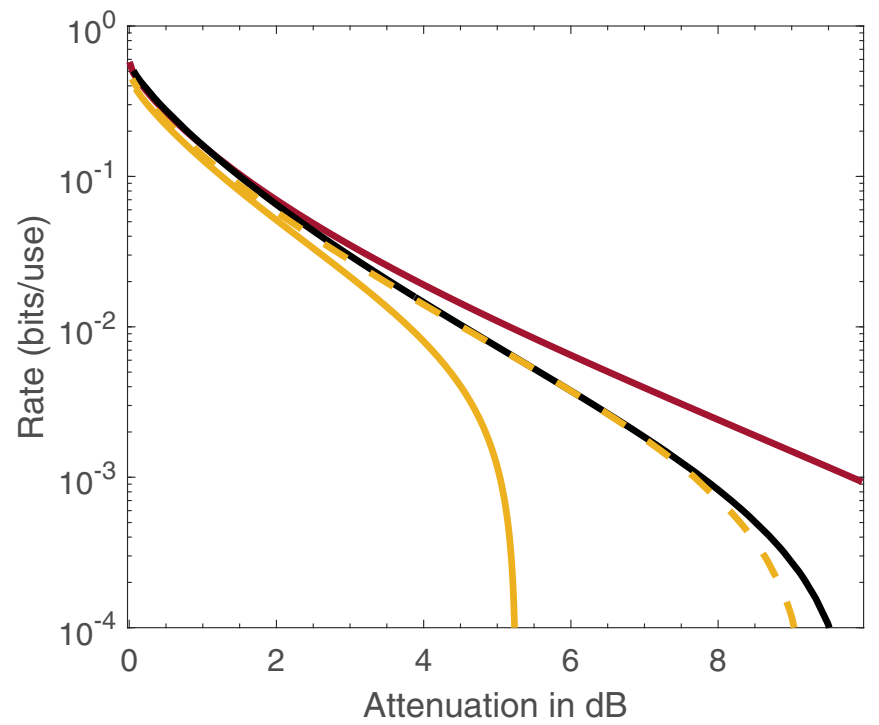

FIG. 4. The secret key rate for $N=2$ and $v_{\text {th }}=0.1$ versus the attenuation in $\mathrm{dB}$. We assume $\alpha=2$ and excess noise $\varepsilon=0.01$. We include the asymptotic case with $\xi=1$ (red solid line) and the composable case, for which we assume $\epsilon_{\mathrm{s}}=\epsilon_{\mathrm{h}}=10^{-10}, \epsilon_{\mathrm{PE}}=10^{-10}$, $p=0.9, \xi=0.99$, and $r=0.01$, for $M=10^{12}$ (yellow dashed line) and $M=10^{9}$ (yellow solid line). For comparison, we also plot the secret key rate assuming coherent states $\left(v_{\text {th }}=0\right.$, black solid line $)$. We observe an advantage when we use preparation trusted noise (compare red and black lines) that can be exploited to mitigate the decrease of the rate in the finite-size regime (similar performance of yellow dashed line and black solid line). All the lines have a truncation accuracy of 14 Fock-basis states.

asymptotic key rate $R$ of the specific protocol under consideration via the transformation in Eq. (41).

Note that, for Gaussian-modulated coherent-state protocols, one can apply a Gaussian de Finetti reduction [62] that enables one to extend the composable security to general coherent attacks. However, this technique does not seem to be applicable to coherent-state protocols with discrete, finite alphabets. Finally, also note that our study involves calculations based on a suitable truncation of the Fock space. The computational cost associated with these calculations is discussed in the Appendix.

\section{ACKNOWLEDGMENTS}

The authors would like to thank Q. Liao and C. Ottaviani for feedback. This work was sponsored by the the European Union via "Continuous Variable Quantum Communications" (CiViQ, Grant Agreement No. 820466) and the EPSRC via the Quantum Communications Hub (Grant No. EP/T001011/1). The computational part of this study was carried out on the Viking Cluster of the University of York.

\section{APPENDIX: COMPUTATIONAL COST}

Here we present some results on the computational cost associated with the calculation of the secret key rate. More precisely, we focus on the time required for the convergence of the entropy of Eve's average state as the number of Fock 


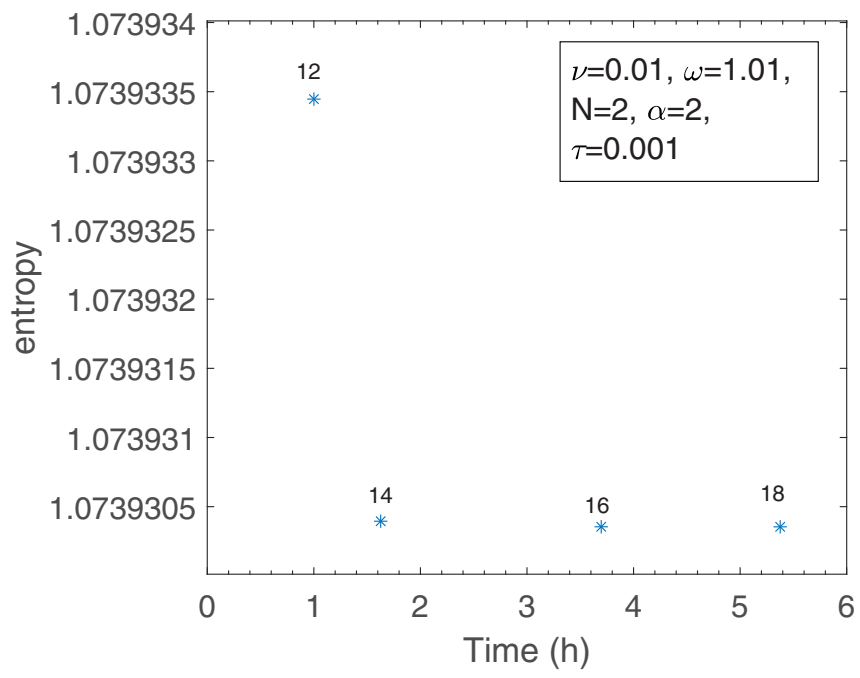

FIG. 5. Entropy convergence versus computational time. Every point has been plotted with a different truncation number in the Fock basis which is indicated on top of the point. As this number is getting larger the entropy is converging to its actual value. For keeping the sixth significant digit, we need to truncate at 14 states, which needs about $1.5 h$. For this plot, we used preparation noise $v=0.01$, channel thermal noise $\omega=1.01$, number of ensemble states $N=2$, amplitude $\alpha=2$ and channel transmissivity $\tau=0.001$.

states increases. This functional is simpler to examine and provides a good estimate of the time needed for the full key rate. Note that, as the transmissivity decreases, i.e., the distance increases, the secret key becomes lower and lower; for this reason, we need more significant digits to approximate it. Correspondingly, this demands extra significant digits for the calculation of the entropy. In Figs. 5-7 we compare this convergence for different values of $\alpha=1,2,3$ while we

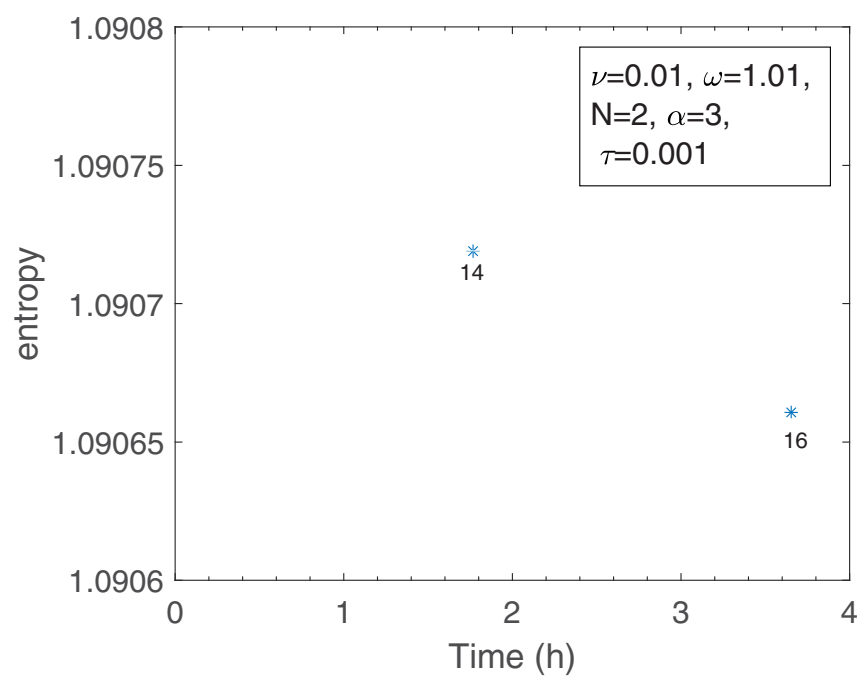

FIG. 6. Entropy convergence versus computational time. Here we present the case of Fig. 5 but for $\alpha=3$. For keeping the fourth significant digit we need to truncate at 16 states or more.

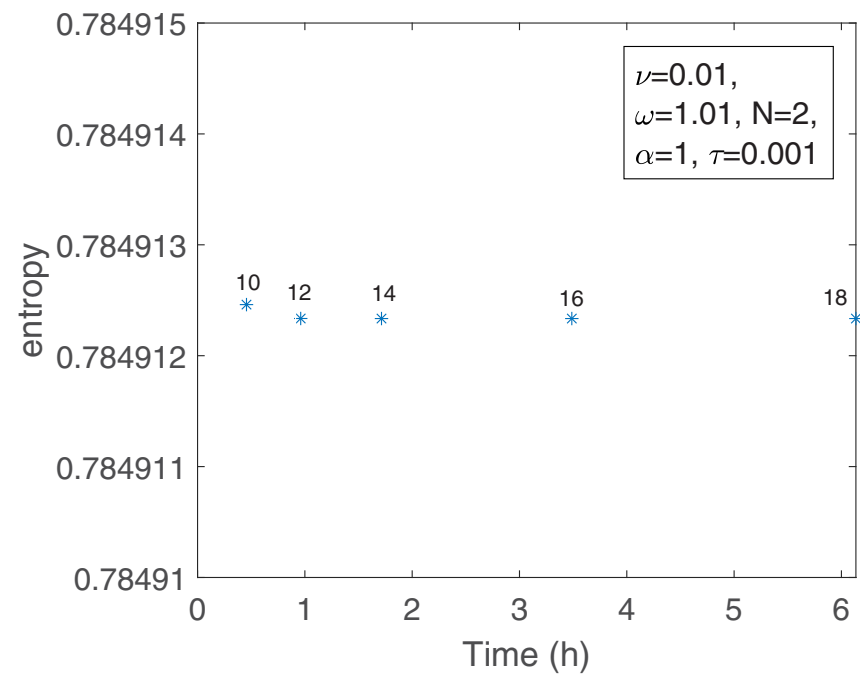

FIG. 7. Entropy convergence versus computational time. For completeness, we present the case of Fig. 5 but for $\alpha=1$, where 10 to 12 states could be enough if we want to keep the fifth significant digit.

set preparation noise $v=0.01$, channel noise $\omega=1.01$, and transmissivity $\tau=0.001$. From Figs. 5, 8, and 9, we may also compare the performances with different values of the transmissivity $\tau=0.001,0.1,0.6$. In Fig. 10 , we present the corresponding case for zero preparation noise. Calculations were performed using MATLAB 2018 on a core of a 20-core 2.0 GHz Intel Xeon 6138 of the Viking cluster [63].

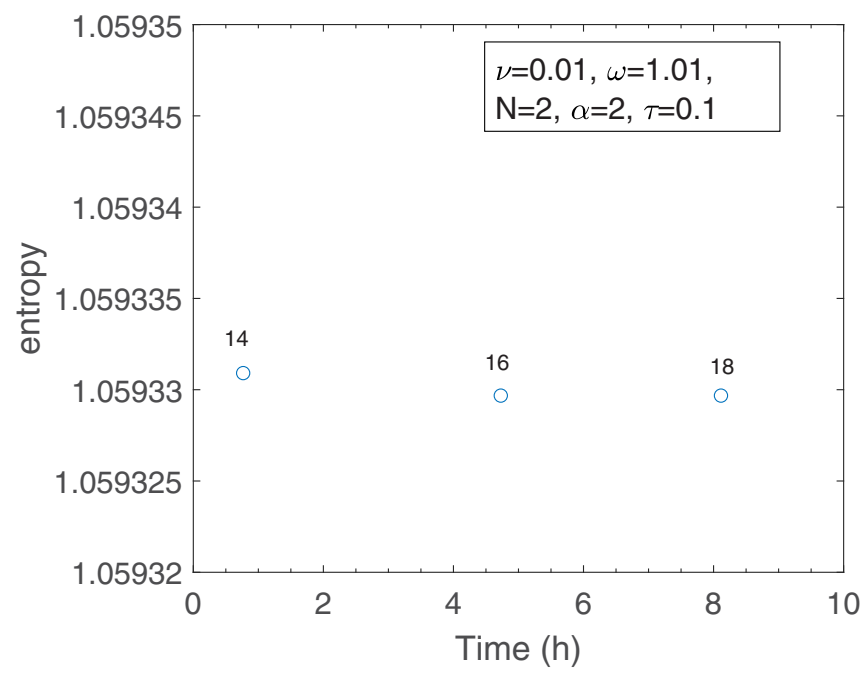

FIG. 8. Entropy convergence versus computational time. We present the case of Fig. 5 but for $\tau=0.1$. Here we need at least 14 states for keeping the fourth significant digit. 


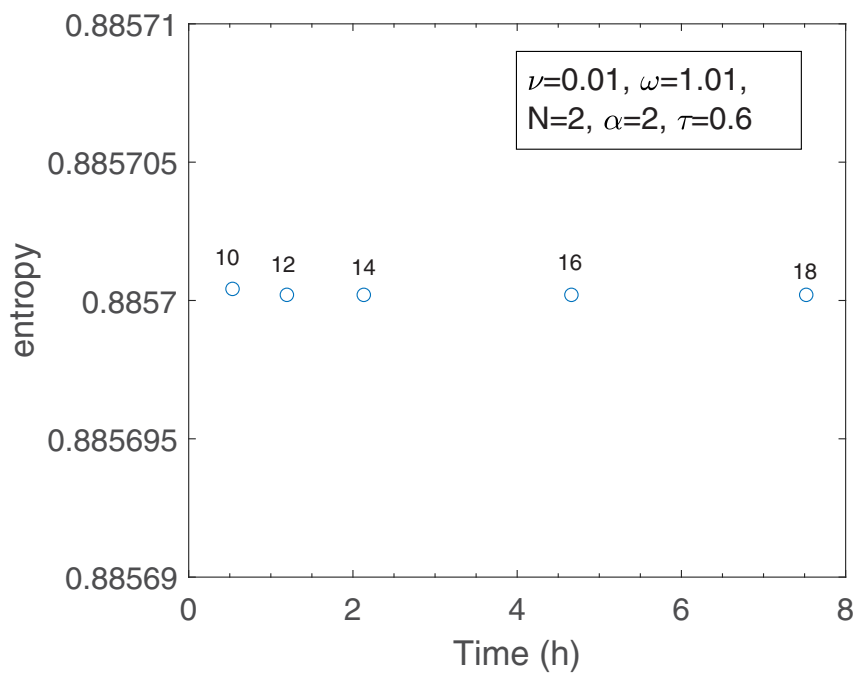

FIG. 9. Entropy convergence versus computational time. We present the case of Fig. 5 but for $\tau=0.6$. We observe that as the transmissivity increases, the convergence becomes faster.

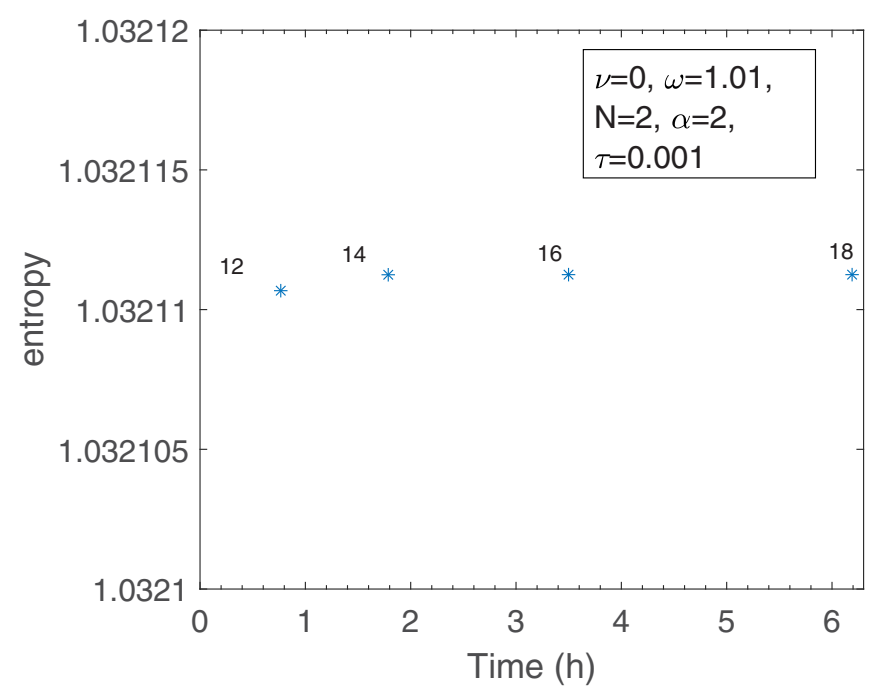

FIG. 10. Entropy convergence versus computational time. For completeness, we present the case of Fig. 5 but for $v=0$.
[1] C. H. Bennett and G. Brassard, Quantum cryptography: Public key distribution and coin tossing, Proceedings of the International Conference on Computers, Systems \& Signal Processing, Bangalore, India, pp. 175-179, December 1984. See also Theor. Comput. Sci. 560, 7 (2014).

[2] A. K. Ekert, Quantum cryptography based on Bell's theorem, Phys. Rev. Lett. 67, 661 (1991).

[3] C. H. Bennett, G. Brassard, and N. D. Mermin, Quantum cryptography without Bell's theorem, Phys. Rev. Lett. 68, 557 (1992).

[4] S. Pirandola, U. L. Andersen, L. Banchi, M. Berta, D. Bunandar, R. Colbeck, D. Englund, T. Gehring, C. Lupo, C. Ottaviani, J. L. Pereira, M. Razavi, J. Shamsul Shaari, M. Tomamichel, V. C. Usenko, G. Vallone, P. Villoresi, and P. Wallden, Advances in quantum cryptography, Adv. Opt. Photon. 12, 1012 (2020).

[5] W. Wootters and W. Zurek, A single quantum cannot be cloned, Nature 299, 802 (1982).

[6] J. Park, The concept of transition in quantum mechanics, Found. Phys. 1, 23 (1970).

[7] S. L. Braunstein and P. van Loock, Quantum information with continuous variables, Rev. Mod. Phys. 77, 513 (2005).

[8] C. Weedbrook, S. Pirandola, R. García-Patrón, N. J. Cerf, T. C. Ralph, J. H. Shapiro, and S. Lloyd, Gaussian quantum information, Rev. Mod. Phys. 84, 621 (2012).

[9] T. C. Ralph, Continuous variable quantum cryptography, Phys. Rev. A 61, 010303 (1999).

[10] M. Hillery, Quantum cryptography with squeezed states, Phys. Rev. A 61, 022309 (2000).

[11] M. D. Reid, Quantum cryptography with a predetermined key, using continuous-variable Einstein-Podolsky-Rosen correlations, Phys. Rev. A 62, 062308 (2000).
[12] N. J. Cerf, M. Levy, and G. Van Assche, Quantum distribution of Gaussian keys using squeezed states, Phys. Rev. A 63, 052311 (2001).

[13] G. Van Assche, J. Cardinal, and N. Cerf, Reconciliation of a quantum-distributed Gaussian key, IEEE Trans. Inf. Theory 50, 394 (2004).

[14] F. Grosshans and P. Grangier, Continuous Variable Quantum Cryptography Using Coherent States, Phys. Rev. Lett. 88, 057902 (2002).

[15] R. García-Patrón, S. Pirandola, S. Lloyd, and J. H. Shapiro, Reverse Coherent Information, Phys. Rev. Lett. 102, 210501 (2009).

[16] S. Pirandola, R. García-Patrón, S. L. Braunstein, and S. Lloyd, Direct and Reverse Secret-Key Capacities of a Quantum Channel, Phys. Rev. Lett. 102, 050503 (2009).

[17] S. Pirandola, R. Laurenza, C. Ottaviani, and L. Banchi, Fundamental limits of repeaterless quantum communications, Nat. Commun. 8, 15043 (2017).

[18] S. Pirandola, S. L. Braunstein, R. Laurenza, C. Ottaviani, T. P. W. Cope, G. Spedalieri, and L. Banchi, Theory of channel simulation and bounds for private communication, Quantum Sci. Technol. 3, 035009 (2018).

[19] R. Filip, Continuous-variable quantum key distribution with noisy coherent states, Phys. Rev. A 77, 022310 (2008).

[20] V. C. Usenko and R. Filip, Feasibility of continuous-variable quantum key distribution with noisy coherent states, Phys. Rev. A 81, 022318 (2010).

[21] C. Weedbrook, S. Pirandola, S. Lloyd, and T. C. Ralph, Quantum Cryptography Approaching the Classical Limit, Phys. Rev. Lett. 105, 110501 (2010).

[22] C. Weedbrook, S. Pirandola, and T. C. Ralph, Continuousvariable quantum key distribution using thermal states, Phys. Rev. A 86, 022318 (2012). 
[23] V. C. Usenko and R. Filip, Trusted Noise in ContinuousVariable Quantum Key Distribution: A Threat and a Defense, Entropy 18, 20 (2016).

[24] P. Papanastasiou, C. Ottaviani, and S. Pirandola, Gaussian oneway thermal quantum cryptography with finite-size effects, Phys. Rev. A 98, 032314 (2018).

[25] S. Pirandola, S. Mancini, S. Lloyd, and S. L. Braunstein, Continuous-variable quantum cryptography using two-way quantum communication, Nat. Phys. 4, 726 (2008).

[26] C. Weedbrook, C. Ottaviani, and S. Pirandola, Two-way quantum cryptography at different wavelengths, Phys. Rev. A 89, 012309 (2014).

[27] V. C. Usenko and F. Grosshans, Unidimensional continuousvariable quantum key distribution, Phys. Rev. A 92, 062337 (2015).

[28] T. Gehring, C. S. Jacobsen, and U. L. Andersen, Singlequadrature continuous-variable quantum key distribution, Quantum Inf. Comput. 16, 1081 (2016).

[29] S. Pirandola, C. Ottaviani, G. Spedalieri, C. Weedbrook, S. L. Braunstein, S. Lloyd, T. Gehring, C. S. Jacobsen, and U. L. Andersen, High-rate measurement-device-independent quantum cryptography, Nat. Photon. 9, 397 (2015).

[30] C. Silberhorn, T. C. Ralph, N. Lütkenhaus, and G. Leuchs, Continuous Variable Quantum Cryptography: Beating the $3 \mathrm{~dB}$ Loss Limit, Phys. Rev. Lett. 89, 167901 (2002).

[31] M. Heid and N. Lütkenhaus, Security of coherent-state quantum cryptography in the presence of Gaussian noise, Phys. Rev. A 76, 022313 (2007).

[32] Y.-B. Zhao, M. Heid, J. Rigas, and N. Lütkenhaus, Asymptotic security of binary modulated continuous-variable quantum key distribution under collective attacks, Phys. Rev. A 79, 012307 (2009).

[33] K. Bradler and C. Weedbrook, Security proof of continuousvariable quantum key distribution using three coherent states, Phys. Rev. A 97, 022310 (2018).

[34] A. Leverrier and P. Grangier, Unconditional Security Proof of Long-Distance Continuous-Variable Quantum Key Distribution with Discrete Modulation, Phys. Rev. Lett. 102, 180504 (2009).

[35] D. Sych and G. Leuchs, Coherent state quantum key distribution with multi letter phase-shift keying, New J. Phys. 12, 053019 (2010).

[36] P. Papanastasiou, C. Lupo, C. Weedbrook, and S. Pirandola, Quantum key distribution with phase-encoded coherent states: Asymptotic security analysis in thermal-loss channels, Phys. Rev. A 98, 012340 (2018).

[37] A. Leverrier and P. Grangier, Continuous-variable quantumkey-distribution protocols with a non-Gaussian modulation, Phys. Rev. A 83, 042312 (2011).

[38] S. Ghorai, P. Grangier, E. Diamanti, and A. Leverrier, Asymptotic Security of Continuous-Variable Quantum Key Distribution with a Discrete Modulation, Phys. Rev. X 9, 021059 (2019).

[39] J. Lin, T. Upadhyaya, and N. Lütkenhaus, Asymptotic Security Analysis of Discrete-Modulated Continuous-Variable Quantum Key Distribution, Phys. Rev. X 9, 041064 (2019).

[40] S. Pirandola, S. L. Braunstein, and S. Lloyd, Characterization of Collective Gaussian Attacks and Security of CoherentState Quantum Cryptography, Phys. Rev. Lett. 101, 200504 (2008).
[41] F. Grosshans, N. J. Cerf, J. Wenger, R. Tualle-Brouri, and P. Grangier, Virtual Entanglement and Reconciliation Protocols for Quantum Cryptography with Continuous Variables, Quantum Inf. Comput. 3(7), 535 (2003).

[42] A. Leverrier, F. Grosshans, and P. Grangier, Finite-size analysis of a continuous-variable quantum key distribution, Phys. Rev. A 81, 062343 (2010).

[43] L. Ruppert, V. C. Usenko, and R. Filip, Long-distance continuous-variable quantum key distribution with efficient channel estimation, Phys. Rev. A 90, 062310 (2014).

[44] S. Pirandola, Limits and security of free-space quantum communications, arXiv:2010.04168.

[45] A. Leverrier, Composable Security Proof for ContinuousVariable Quantum Key Distribution with Coherent States, Phys. Rev. Lett. 114, 070501 (2015).

[46] C. Lupo, C. Ottaviani, P. Papanastasiou, and S. Pirandola, Continuous-variable measurement-device-independent quantum key distribution: Composable security against coherent attacks, Phys. Rev. A 97, 052327 (2018).

[47] M. Tomamichel, C. Schaffner, A. Smith, and R. Renner, Leftover Hashing Against Quantum Side Information, IEEE Trans. Inf. Theory 57, 5524 (2011).

[48] M. Tomamichel, Ph.D. thesis, Swiss Federal Institute of Technology (ETH), Zurich, 2012, arXiv:1203.2142.

[49] M. Tomamichel, R. Colbeck, and R. Renner, A Fully Quantum Asymptotic Equipartition Property, IEEE Trans. Inf. Theory 55, 5840-5847 (2009).

[50] C. Portmann and R. Renner, Cryptographic security of quantum key distribution, arXiv:1409.3525v1.

[51] G. Giedke and J. I. Cirac, Characterization of Gaussian operations and distillation of Gaussian states, Phys. Rev. A 66, 032316 (2002).

[52] J. Eisert and M. Plenio, Introduction to the basics of entanglement theory in continuous-variable systems, Int. J. Quantum. Inform. 1, 479 (2003).

[53] S. Pirandola, G. Spedalieri, S. L. Braunstein, N. J. Cerf, and S. Lloyd, Optimality of Gaussian Discord, Phys. Rev. Lett. 113, 140405 (2014).

[54] This state is calculated by using [[4], Eq. (A7)] replacing its mean and CM from Eqs. (6) and (7) and expressing the quadrature operators in terms of the Fock basis considering the appropriate truncation.

[55] I. Devetak, The private classical capacity and quantum capacity of a quantum channel, IEEE Trans. Inf. Theory 51, 44 (2005).

[56] Y. Zhang, Z. Chen, S. Pirandola, X. Wang, C. Zhou, B. Chu, Y. Zhao, B. Xu, S. Yu, and H. Guo, Long-Distance ContinuousVariable Quantum Key Distribution over $202.81 \mathrm{~km}$ of Fiber, Phys. Rev. Lett. 125, 010502 (2020).

[57] The analytical dependence of $\operatorname{leak}_{\mathrm{EC}}\left(n, \epsilon_{\mathrm{cor}}\right)$ on $\epsilon_{\mathrm{cor}}$ is determined in an experimental scenario after the choice of the linear EC code. At that point, one can more precisely relate $\xi$ to $\epsilon_{\text {cor }}$ through Eq. (49). From typical experiments for Gaussian protocols, it is known that $\xi=0.95[58]$ and $\xi=0.98$ [[59,60]] is achievable. In the case of discrete-alphabet encoding the EC is very efficient. This is why we chose a reconciliation efficiency equal to $\xi=0.99$.

[58] P. Jouguet, D. Elkouss, and S. Kunz-Jacques, High-bit-rate continuous-variable quantum key distribution, Phys. Rev. A 90, 042329 (2014). 
[59] M. Milicevic, C. Feng, L. M. Zhang, and P. G. Gulak, Quasi-cyclic multi-edge LDPC codes for longdistance quantum cryptography, npj Quant. Info. 4, 21 (2018).

[60] X. Wang, Y.-C. Zhang, Z. Li, B. Xu, S. Yu, and H. Guo, Efficient rate-adaptive reconciliation for continuous-variable quantum key distribution, Quantum Inf. Comput. 17, 1123 (2017).
[61] L. Banchi, S. L. Braunstein, and S. Pirandola, Quantum Fidelity for Arbitrary Gaussian States, Phys. Rev. Lett. 115, 260501 (2015).

[62] A. Leverrier, Security of Continuous-Variable Quantum Key Distribution via a Gaussian de Finetti Reduction, Phys. Rev. Lett. 118, 200501 (2017).

[63] https://wiki.york.ac.uk/display/RHPC/Viking+-+The+York+ Super+Advanced+Research+Computing+Cluster 